\title{
EDITORIAL
}

\section{Gene therapy for pain: Introduction to the special issue}

\section{Gene Therapy (2009) 16, 453-454; doi:10.1038/gt.2009.18}

Gene therapy was first proposed as an approach to the treatment of recessive inherited disease, in which addition of a correct copy of the mutant gene would restore normal function. Although heuristically attractive, such applications of gene therapy have proven to be a high bar and with few exceptions the focus of gene therapy has moved to approaches designed to ameliorate acquired diseases processes. Chronic pain is an example of a disease process affecting a large proportion of the population for which effective long-term treatments have remained elusive. Recent studies of the mechanisms that underlie the development of chronic pain have created a base of physiological and genetic information that identify multiple points of intervention to treat chronic pain. On the basis of these insights, gene therapy may be used to produce products that block pain transmission or reverse the chronic pain state. In the review chapters provided in this special issue, the most current methods and strategies for treatment of chronic pain are described, which support the development of gene therapy as a potentially practical approach suitable for intervening in the treatment of intractable pain.

Pain signals are transmitted to the brain through a relatively well-defined neuroanatomic pathway (Figure 1), but the anatomic specificity belies an underlying commonality; each of the neurotransmitters, receptors, ion channels and signaling molecules involved in nociceptive neurotransmission from the periphery to the brain are employed by the nervous system in pathways subserving other functions, and in a substantial number of cases in organs outside the nervous system. As a result, systemic drugs administered to reduce pain often produce 'off-target' effects that preclude their use in the full pain-relieving dose.

Traditional pharmaceutical and academic research has focused on the search for small molecules that might selectively relieve pain, and for earlier undiscovered components involved in the pathogenesis or neurotransmission of pain signals that might serve as novel targets for small molecules. Gene therapy for pain is based on the premise that focal delivery of peptides targeting wellknown and understood elements of the pain pathway might be used to selectively interrupt nociceptive neurotransmission by the anatomic specificity achieved through gene delivery, and can be extended to the use of genes that inhibit inappropriate nociceptor activation and firing. The preclinical animal studies reviewed in this volume include the use of plasmids injected intrathecally to transduce meninges and release a transgene product into the cerebrospinal fluid, lentiviral vectors injected directly into the dorsal horn of the spinal cord, and the use of either adeno-associated virus injected intrathecally or herpes simplex virus (HSV) injected peripherally to transduce neurons of the dorsal root ganglia. These preclinical studies span the full range of chronic pain including: inflammatory somatic pain, visceral pain, neuropathic pain and pain caused by cancer.

Two aspects of these reports bear noting. The first is the broad range of gene products that have been studied in models of pain. The earliest gene transfer experiments used gene transfer to express inhibitory neurotransmitters ( $\beta$-endorphin, enkephalin) and the human trial currently in progress will examine the safety of an HSV vector-expressing enkephalin in patients with pain from cancer. But concurrent with increased understanding of the pathophysiology of pain in the nervous system, the range of gene transfer targets has expanded substantially. Reports reviewed in this volume include the expression of anti-inflammatory peptides (Interleukin (IL)-4, IL-10 and the soluble p55 tumor necrosis factor receptor) in cerebrospinal fluid or dorsal root ganglia, NF- $\mathrm{kB}$ in glia in the dorsal horn of spinal cord, and calcitonin generelated peptides and voltage-gated sodium channels in neurons of the dorsal root ganglia. Local expression of opioid receptors and of the glycine receptor has been shown to enhance the pain-relieving effects of drugs.

The second aspect is the emergence of HSV as a gene transfer vector. Although often relegated to the bottom of the list of potential gene transfer vectors, five of the eight chapters in this volume involve the use of HSV-mediated gene transfer. HSV suffers in comparison to other vectors because its $150 \mathrm{~kb}$ genome is relatively difficult to manipulate, requiring homologous recombination and selection rather than cloning to produce new vectors, though recent advances in the creation of HSV vectors grown and manipulated in bacteria may help to overcome this obstacle. But the natural neurotropism of the

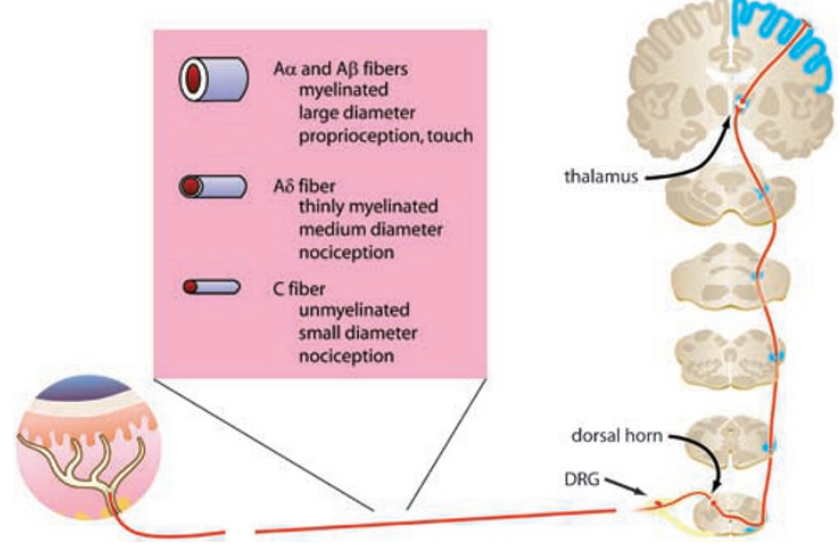

Figure 1 Neuroanatomy of pain. Acute pain signals are transmitted by $\mathrm{A} \delta$ and $\mathrm{C}$ fibers to the spinal cord. In the dorsal horn of spinal cord the primary (dorsal root ganglia) afferent synapses with second-order neurons that project rostrally. Third-order neurons of the thalamus and brainstem nuclei project to sensory and limbic cortex. 
parental wild-type virus, along with its natural propensity to establish a life-long persistent state as an intranuclear (but non-integrating) element in neurons makes vectors derived from HSV particularly suitable for nervous system applications. Oncolytic HSV have been tested safely in several different tumors (breast, brain) in patients, and in the human trial reported in the final review of this group investigators have begun to examine the safety of non-replicating HSV as a gene transfer vector in patients with pain from cancer.

Chronic pain is complex, and there are significant limitations to what animal studies can tell us about novel treatments. But with the first human trial currently underway we are at the threshold of being able to test the effectiveness of these vectors and transgene products in patients with different types of chronic pain, a process that will take us one step further toward the development of gene therapy for pain.

\section{Acknowledgements}

This work was supported by NIH grants NS044507, NS038850, DK044935, grants from the Department of Veterans Affairs and the Juvenile Diabetes Research Foundation.

JC Glorioso ${ }^{1}$ and DJ Fink ${ }^{2}$ ${ }^{1}$ University of Pittsburgh, Pittsburgh, PA, USA and ${ }^{2}$ Department of Neurology, University of Michigan and VA Ann Arbor Healthcare System, Ann Arbor, MI, USA 